\title{
Ensayo del material compuesto poli-eter-eter-cetona/ fibra de carbono en el acetábulo de prótesis totales de cadera
}

FERNÁNDEZ FAIREN M., POLINENI V. K., WANG A., SALA A.

Fundación Policlínica del Vallés, Granollers. Barcelona.

\section{Introducción}

Los avances en materiales, diseños y técnicas han permitido que en la actualidad pueda esperarse una supervivencia estimable de las prótesis totales de cadera $(1,2)$. De cualquier manera, aproximadamente un $10 \%$ de todos los procedimientos protésicos realizados anualmente son recambios de prótesis que han fallado, estando el desgaste en la base una buena parte de esos fallos (3). Y ésto es aún más acusado en el grupo de pacientes de edad menor a 40 años. Efectivamente, aunque todos los materiales que se utilizan hoy día en la confección de prótesis se desgastan en mayor o menor medida en todos los casos, lo hacen de manera especialmente acelerada y agresiva en pacientes jóvenes y activos $(4,5,6)$.

El desgaste atenta directamente contra la integridad morfológica y funcional de los implantes. Pero es que, sobre todo, las partículas así producidas son capaces de provocar una reacción inflamatoria a cuerpo extraño $(7,8)$ que causa la reabsorción del hueso alrededor del implante (9), lo que se ha dado en llamar osteólisis y reconocido como un grave problema que compromete la supervivencia a largo plazo de las prótesis (10). Se ha comprobado una relación directa entre magnitud del desgaste y reabsorción del calcar $(11,12)$, o entre aquella y la incidencia de aflojamiento radiológico $(13,14)$.

La preocupación por evitar los efectos perniciosos del desgaste de las superficies articulares han llevado a lo largo de toda la historia de la cirugía protésica a buscar soluciones optimizando los materiales empleados. El polimetilmetacrilato utilizado por Judet a finales de los 30s y los 40s (15) dió unos resultados catastróficos, al igual que el politetrafluoroetileno al que recurrió inicialmente Charnley. El polietileno de extra alto peso molecular que

Correspondencia:

M. Fernández Fairen

Río de Oro, 37, Barcelona-08034.

Tel. 934870641. Fax: 934883590.

E-mail: axisbiomec@bsab.com comenzó a implantar en 1962 en el acetábulo (16), en oposición a un componente femoral metálico, es el superviviente hoy día, a pesar de haber sido estudiados y ensayados múltiples materiales (17) y haberlo intentado en clínica con el poliacetal $(18,19)$ y el poliester (20). La mejora supuesta por el tratamiento de la superficie metálica de la cabeza femoral mediante implantación iónica o recubrimiento con capas de pasivación es controvertida $(21,22,23)$. En ese mismo sentido hay que considerar la propuesta de usar un par metal-metal $(24,25)$ o cerámica-cerámica, con una discordancia en los resultados obtenidos $(26,27,28,29)$.

Otra vía ha sido la búsqueda de disminuir el desgaste del polietileno aumentando su cristalinidad $(30,31)$, pero hasta la fecha no ha sido posible demostrar esa ventaja en el campo clínico (31). Lo mismo sucede con el material compuesto, o composite, de poli-etileno y fibras de carbono, o Poly II $(32,33)$. Hemos intentado obviar esos inconvenientes modificando la fase polimérica, o matriz, del compuesto, y en la experimentación de ese producto consiste este trabajo.

\section{Material y método}

Se fabricaron mediante inyección en molde, componentes acetabulares de $28 \mathrm{~mm}$ de diámetro, según diseño Sistema $12^{\mathrm{TM}}$ Howmedica Inc, talla P-3, neutros, de un material compuesto a base de $70 \%$ de poli-eter-eter-cetona (PEEK) (ICI, grado $150 \mathrm{G})$, y $30 \%$ de fibra de carbono grafítico troceado (Amo- co, grado VMX-12) (34). Las propiedades mecánicas de tal compuesto se expresan en la tabla I. El comportamiento frente al desgaste de los mismos se comparó con el de componentes de polietileno de peso molecular ultra alto (UHMWPE) (Sistema $12^{\mathrm{TM}}$ Howmedica Inc, talla P-3, neutros, Cat \# : 5260-4-372, Lote \# : UMZMA 9608688). Estos últimos tenían en el dorso 12 relieves de retención para quedar sujetos a una cúpula acetabular metálica de cromo-cobalto-molibdeno (Vitallium, Howmedica Inc, Cat \# : 6302-1-050, Lote \# : 
CRFMB, talla P-3, $50 \mathrm{~mm}$ ). Los elementos de compuesto tenían 8 relieves ahusados de retención y se aseguraban en cúpulas idénticas a las citadas mediante una junta constituida por un anillo exagonal abierto de PEEK (fig 1). La fuerza de inserción necesaria para colocar los elementos de compuesto fue de $518 \mathrm{~N}$ de promedio, y de $580 \mathrm{~N}$ para los de UHMWPE. La fuerza precisa para desensamblar los componentes cotiloideos articulares del interior de los respaldos metálicos oscilaron entre 1018 y $1497 \mathrm{~N}$ en el caso de los primeros, y entre 856 y $1117 \mathrm{~N}$ para los segundos.

Ambos tipos se esterilizaron por radiación gamma a 2,5 Mrad (25 kGg), empleándose en este estudio 5 implantes de material compuesto y 3 de UHMWPE.

Se les opusieron en el simulador cabezas femorales de $28 \mathrm{~mm}$ de zirconia (Zircone Prozyr, Howmedica BG, Ref \# : 4653-40, Lote \# : 76398/ 62801), y se utilizó como lubricante suero bovino obtenido de Hyclone Laboratories (Cat \# : SH30212.05) con un contenido, cuidadosamente controlado, de $41 \mathrm{~g} / \mathrm{l}$ de proteínas y partículas inorgánicas. Esto ayuda a mantener la consistencia del lubricante durante los ensayos minimizando su variabilidad. Se añadió al suero ácido etilen-diaminotetra-acético (EDTA) pH estabilizado, para impedir la formación de cualquier precipitado sobre la cabeza femoral y para retardar la descomposición del suero. Este fue filtrado antes de su empleo a través de filtros de $0,2 \mu \mathrm{m}$ para eliminar cualquier partícula por encima de ese tamaño.Las pruebas de desgaste se realizaron en un simulador de cadera MTS, de 8 unidades de ensayo (35).

Cada unidad constaba de los elementos de sujección de cabeza y cotilo, alineados según el mismo eje vertical. La espiga femoral tenía un cono adecuado a la cabeza. El conjunto se encontraba colocado dentro de una cámara de acrílico, para contener el suero bovino utilizado en la lubricación de la articulación , con una base de acero inoxidable y bloqueada sobre un bloque inclinado $23^{\circ}$.

Los ensayos se realizaron siguiendo la disposi- ción anatómica, es decir el cotilo situado arriba y la cabeza femoral distalmente. El bloque inclinado gira a una velocidad de $1 \mathrm{~Hz}$ y se aplicó una fuerza axial de compresión con una carga mínima/máxima de 50/2450 N, de carácter cíclico y según el perfil definido por Paul (36). Se siguieron protocolos standard para ensayos, para lavar, pesar y determinar el desgaste de los implantes (37).

Una máquina de medida de coordenadas (CMM) leyó las superficies articulares a los 0 ciclos, y a 1 , $3,5,8$ y 10 millones de ciclos. El cambio volumétrico y lineal de los componentes fué calculado a partir de la lectura de la red de coordenadas medidas sobre las superficies articulares. También se midió en los mismos intervalos la pérdida de peso por desgaste de los implantes de UHMWPE y a 0 ciclos y a 10 millones de ciclos los de compuesto, para evitar la lesión de los elementos insertados al soltar repetidamente el anillo de retención. Los valores de peso perdido se convirtieron en volumen perdido usando las densidades de los respectivos materiales $(\mathrm{UHMWPE}=0,935 \mathrm{~g} / \mathrm{cc}$; compuesto $=1,5$ $\mathrm{g} / \mathrm{cc}$ ). Se hicieron controles por inmersión en agua desionizada, a temperatura ambiente, durante las interrupciones del test, para minimizar el error producido por la posible absorción de líquido por parte de los materiales experimentados.

Además de todo ésto, se hizo una espectroscopia por infrarrojos y una cromatografía por permeación en gel para comprobar si se producían alteraciones químicas en el material durante el proceso de fabricación, y toda una serie de estudios de biocompatibilidad del compuesto y de sus componentes, en masa, en partículas y en disolución. Se realizaron test de mutageneidad (ames, L5178Y, aberraciones cromosómicas, transformación BALB/c3T3, síntesis de DNA en células hepáticas murinas in vitro). Se practicaron test de toxicidad y carcino-geneidad observando el efecto de un extracto del material y del material mismo en fibroblastos de ratones L-929, la toxicidad local en conejos seguidos entre 0 y 72 horas tras inoculación intradérmica de un extracto del producto, la

Tabla I. Propiedades mecánicas del material compuesto utilizado.

\begin{tabular}{|l|c|c|}
\hline Propiedades mecánicas & PEEK & 30\% FC+PEEK \\
\hline Resistencia en tracción $(\mathrm{MPa})$ & 97 & 224 \\
\hline Resistencia en flexión $(\mathrm{MPa})$ & 170 & 355 \\
\hline Resistencia en compresión $(\mathrm{MPa})$ & 118 & 240 \\
\hline Resistencia al cizallamiento $(\mathrm{MPa})$ & 53 & 97 \\
\hline
\end{tabular}


toxicidad sistémica en ratones entre 0 y 72 horas de la inyección del extracto, y la toxicidad y carcinógenesis a 2 años en ratas Sprage-Dawley.

Se llevaron a cabo un test de pirógenos registrando las temperaturas de conejos hasta 3 horas después de esa inyección, un test de hemolisis de sangre de conejo directamente con el producto y con extracto salino del mismo, y una sensibilización de cobayas inyectados primariamente en intradérmico con un extracto del material y observados luego de 0 a 96 horas después de una segunda aplicación del mismo.

Por último, se analizaron las respuestas macro y microscópicas 90 días postimplantación del material fragmentado en músculo de conejo, a las 52 semanas del implante en hueso de conejos y a los 2 años de colocación de un vástago endomedular en perro, y la histología de los tejidos circundantes a una prótesis de cadera en servicio en perro durante 7 años.

\section{Resultados}

El desgaste fue pues evaluado a partir de la pérdida de peso de los especímenes tras servicio y de los cambios de volumen calculados desde las lecturas CMM. Después de 10 millones de ciclos, los componentes de UHMWPE mostraron un desgas- te volumétrico total de $353,73 \pm 54.36 \mathrm{~mm}^{3}$, mientras que para los del material compuesto fué de $3,91 \pm 0,90^{3}$ (fig 1 y tabla II). Eso indica claramente que el desgaste de los cotilos de compuesto es aproximadamente sólo el $1 \%$ del de los de UHMWPE convencional. Debido a la baja tasa de desgaste, la desviación standard para los elementos de compuesto es de aproximadamente $25 \%$, en tanto que para los de UHMWPE es del 15\%. Dada la naturaleza altamente aleatoria del proceso, se consideran generalmente aceptables desviaciones de hasta el 30\% en ensa yos similares en simulador.

La tabla III muestra los resultados obtenidos a partir del método CMM. Es evidente que los cotilos de material compuesto demuestran de nuevo una resistencia superior al desgaste. El desgaste es tan bajo que las lecturas CMM dan valores negativos en los cambios de volumen, indicando que éstos están fuera de la capacidad de resolución de la máquina. Por otra parte, los resultados obtenidos con este sistema para los componentes de UHMWPE exhiben una excelente concordancia con los observados en los mismos según el método gravimétrico anteriormente expresado.

La CMM facilita también la estimación del desgaste lineal, es decir la penetración de la cabeza en el componente acetabular. La figura 2 y la tabla IV

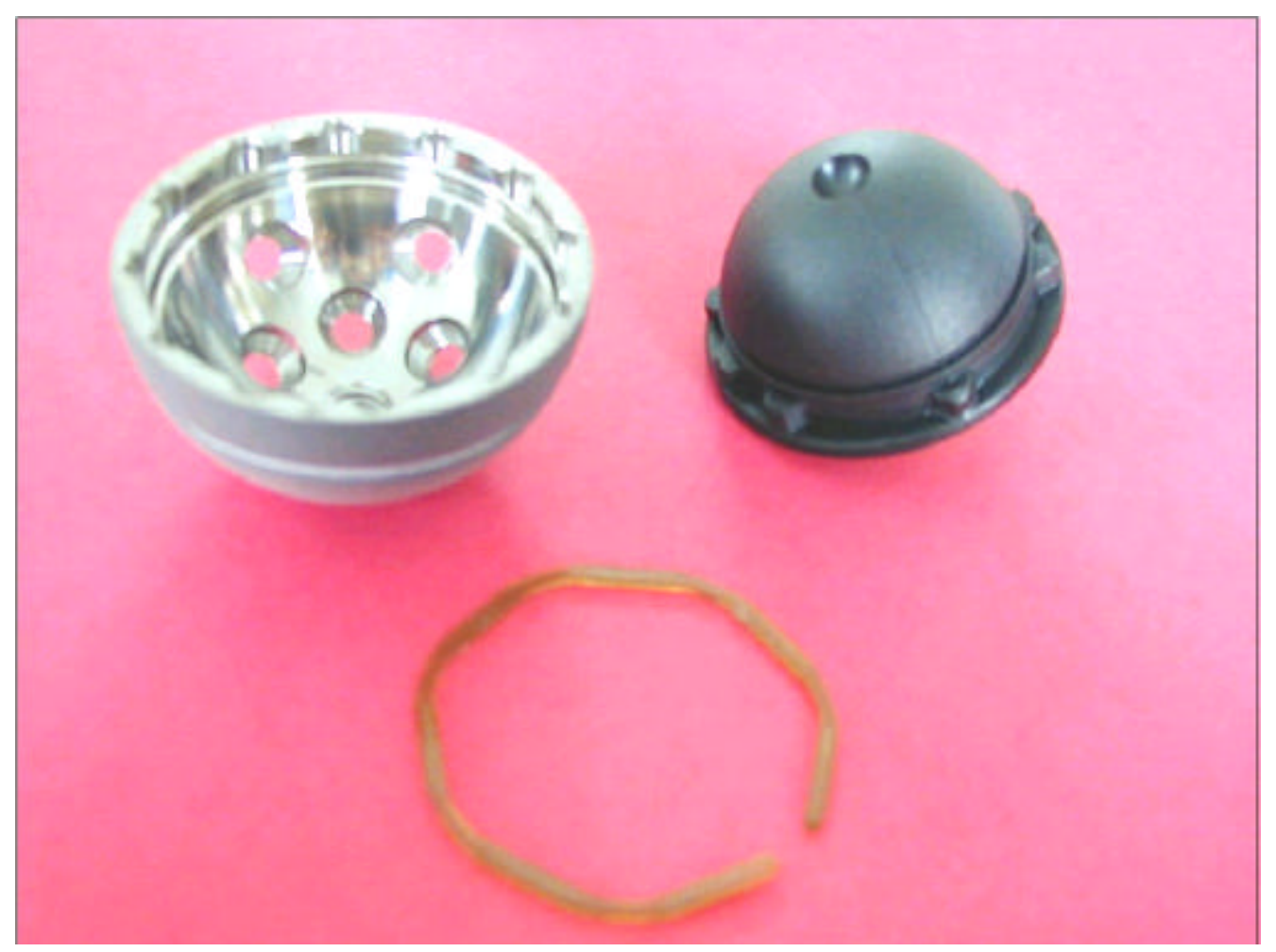

Figura 1. Inserto de PEEK/fibra de C 
recogen esos datos. Los cotilos de UHMWPE sufren una penetración lineal de $66,41 \pm 12,64 \mu \mathrm{m}$ por millón de ciclos, en tanto que ese rango era de sólo
$1,72 \pm 1,36 \mu \mathrm{m}$ para los de compuesto. La alta desviación standard de estos últimos indica de nuevo que las modificaciones son demasiado pequeñas

Tabla II. Desgaste volumétrico de los componentes acetabulares a partir del método gravimétrico.

\begin{tabular}{|c|c|c|c|c|c|}
\hline $\begin{array}{l}\text { Desgaste individual } \\
\qquad\left(\mathrm{mm}^{3}\right)\end{array}$ & 1 & 3 & 5 & 8 & 10 \\
\hline $\mathrm{n}^{\circ} 1$ UHMWPE & 63,307 & 145,797 & 243,501 & 340,381 & 391,071 \\
\hline $\mathrm{n}^{\circ} 2$ UHMWPE & 57,069 & 134,189 & 229,226 & 322,984 & 378,748 \\
\hline $\mathrm{n}^{\circ} 3$ UHMWPE & 57,619 & 130,988 & 196,374 & 254,815 & 291,362 \\
\hline $\mathrm{n}^{\circ} 4 \mathrm{FC} / \mathrm{PEEK}$ & & & & & 2,589 \\
\hline $\mathrm{n}^{\circ} 5$ FC/PEEK & & & & & 3,819 \\
\hline $\mathrm{n}^{\circ} 6 \mathrm{FC} / \mathrm{PEEK}$ & & & & & 3,735 \\
\hline $\mathrm{n}^{\circ} 7$ FC/PEEK & & & & & 4,399 \\
\hline $\mathrm{n}^{\circ} 8$ FC/PEEK & & & & & 5,015 \\
\hline $\begin{array}{c}\text { Promedio y desviación } \\
\text { standard }\end{array}$ & & & & & \\
\hline \multirow[t]{2}{*}{ UHMWPE } & 59,33 & 136,99 & 223,03 & 306,06 & 353,73 \\
\hline & $\pm 3,45$ & $\pm 7,79$ & $\pm 24,17$ & $\pm 45,22$ & $\pm 54,36$ \\
\hline \multirow[t]{2}{*}{ FC/PEEK } & & & & & 3,911 \\
\hline & & & & & $\pm 0,90$ \\
\hline
\end{tabular}

Tabla III. Desgaste volumétrico a partir del método CMM.

\begin{tabular}{|c|c|c|c|c|c|}
\hline $\begin{array}{c}\text { Desgaste individual } \\
(\mathbf{m m})\end{array}$ & 1 & 3 & 5 & 8 & 10 \\
\hline $\mathrm{n}^{\circ} 1$ UHMWPE & 81,23 & 173,93 & 256,13 & 369,72 & 399,48 \\
\hline $\mathrm{n}^{\circ} 2$ UHMWPE & 80,59 & 162,89 & 244,68 & 353,75 & 393,14 \\
\hline $\mathrm{n}^{\circ} 3$ UHMWPE & 89,29 & 145,24 & 204,08 & 289,13 & 307,99 \\
\hline $\mathrm{n}^{\circ} 4 \mathrm{FC} / \mathrm{PEEK}$ & $-11,14$ & 22,61 & $-16,03$ & $-35,23$ & $-33,97$ \\
\hline $\mathrm{n}^{\circ} 5$ FC/PEEK & $-16,63$ & $-12,98$ & $-9,18$ & $-30,23$ & $-24,27$ \\
\hline $\mathrm{n}^{\circ} 6$ FC/PEEK & $-17,14$ & $-16,99$ & $-8,85$ & $-2,34$ & $-37,36$ \\
\hline $\mathrm{n}^{\circ} 7$ FC/PEEK & $-8,59$ & $-19,71$ & $-9,28$ & -3141 & $-20,32$ \\
\hline $\mathrm{n}^{\circ} 8$ FC/PEEK & $-14,55$ & $-24,07$ & $-17,52$ & $-4,98$ & $-31,91$ \\
\hline \multicolumn{6}{|l|}{$\begin{array}{c}\text { Promedio y desviación } \\
\text { standard }\end{array}$} \\
\hline \multirow[t]{2}{*}{ UHMWPE } & 83,71 & 160,69 & 234,96 & 337,54 & 366,87 \\
\hline & $\pm 4,85$ & $\pm 14,47$ & $\pm 27,35$ & $\pm 42,67$ & $\pm 51,09$ \\
\hline \multirow[t]{2}{*}{ FC/PEEK } & 13,61 & $-19,27$ & 12,17 & $-20,84$ & $-29,57$ \\
\hline & $\pm 3,67$ & $\pm 4,45$ & $\pm 4,24$ & $\pm 15,82$ & $\pm 7,06$ \\
\hline
\end{tabular}


para ser apreciadas por la máquina.

Durante los ensayos el suero que circundaba el par cotilo de material compuesto/cabeza de zirconia se oscureció durante el primer 0,5 millón de ciclos. Este oscurecimiento no se hizo ostensible más allá de ese punto. Se encontraron adheridos entre el reborde del cotilo de compuesto y el componente metálico pequeñas partículas y filamentos, seguramente generados durante el asentamiento inicial de ambos elementos. Después de esos primeros ciclos, y totalmente efectuado ese asentamiento, debe quedar eliminado el rozamiento entre ellos. El mecanismo de bloqueo constituido por el anillo de
PEEK probó ser eficaz y durable a satisfacción. No se detecto ninguna movilidad entre las dos piezas del cotilo. El examen visual del elemento insertado de material compuesto mostró después del ensayo cierto grado de pulido en los relieves de engarce con el metal. A nivel de las cabezas de zirconia no se detectaron nunca marcas o erosiones.

Respecto a la verificación de la estabilidad química del compuesto a través del proceso de fabricación quedó demostrada a satisfacción en la cromatografía, no evidenciándose ningún cuerpo orgánico extraño en la espectroscopia por IR.

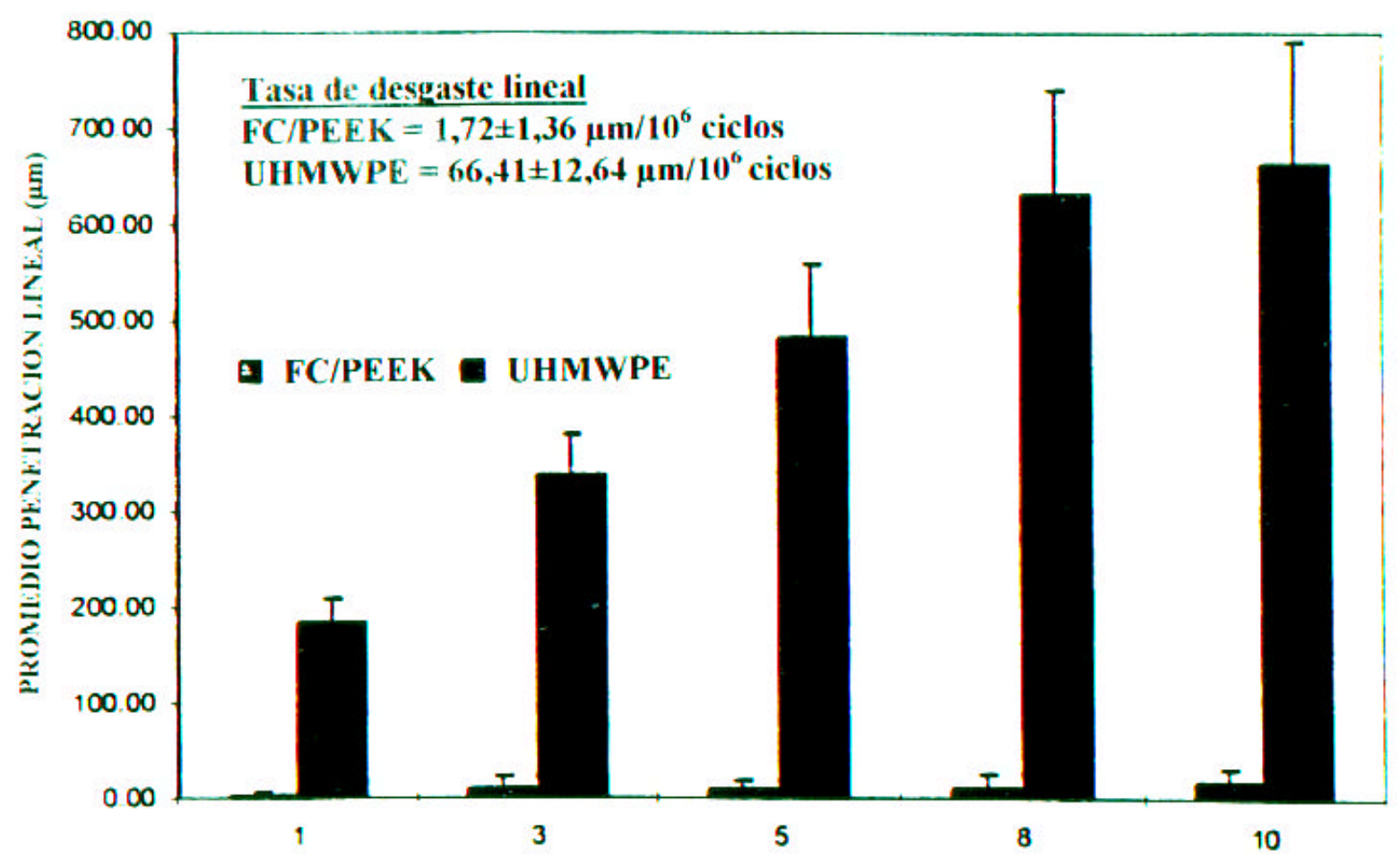

NI NIRO DF CICLOS (millones)

Figura 2. Desgaste volumétrico de componentes de UHMWPE a lo largo de la experimentación y del material compuesto FC/PEEK tras 10 millones de ciclos en el simulador.

Tabla IV. Penetración lineal ( $\mu \mathrm{m})$

\begin{tabular}{|c|c|c|c|c|c|}
\hline & \multicolumn{5}{|c|}{ Millones de ciclos } \\
\hline $\begin{array}{c}\text { Promedio y } \\
\text { desviación standard }\end{array}$ & $\mathbf{1}$ & $\mathbf{3}$ & $\mathbf{5}$ & $\mathbf{8}$ & $\mathbf{1 0}$ \\
\hline UHMWPE & 183,64 & 337,65 & 481,67 & 632,12 & 664,13 \\
\hline & $\pm 24,15$ & $\pm 42,63$ & $\pm 77,16$ & $\pm 108,41$ & $\pm 126,38$ \\
\hline FC/PEEK & 0,71 & 9,19 & 9,14 & 10,67 & 17,17 \\
\hline & $\pm 5,21$ & $\pm 13,14$ & $\pm 9,23$ & $\pm 14,09$ & $\pm 13,59$ \\
\hline
\end{tabular}


En cuanto a la investigación de la biocompatibilidad del compuesto, no se manifestaron mutaciones o aberraciones cromosómicas, ni lisis celular, ni irritación ni toxicidad local, ni mortalidad o evidencia de toxicidad sistémica. Tampoco se consideró pirógeno, ni causó hemolisis, ni hubo sensibilización dérmica. Los análisis histológicos de los tejidos en los que fué implantado el material o los tejidos periarticulares sometidos a la presencia de partículas provenientes del desgaste de las superficies protésicas no mostraron reacciones macros- cópicas significativamente diferentes a controles, y a nivel microscópico el material fué clasificado como no irritante.

\section{Discusión}

Los materiales compuestos (composites) son materiales multifásicos constituidos por una matriz y un relleno de la misma por fibras. Los materiales correspondientes a una y a otras suelen ser complementarios en cuanto a sus propiedades, produ-
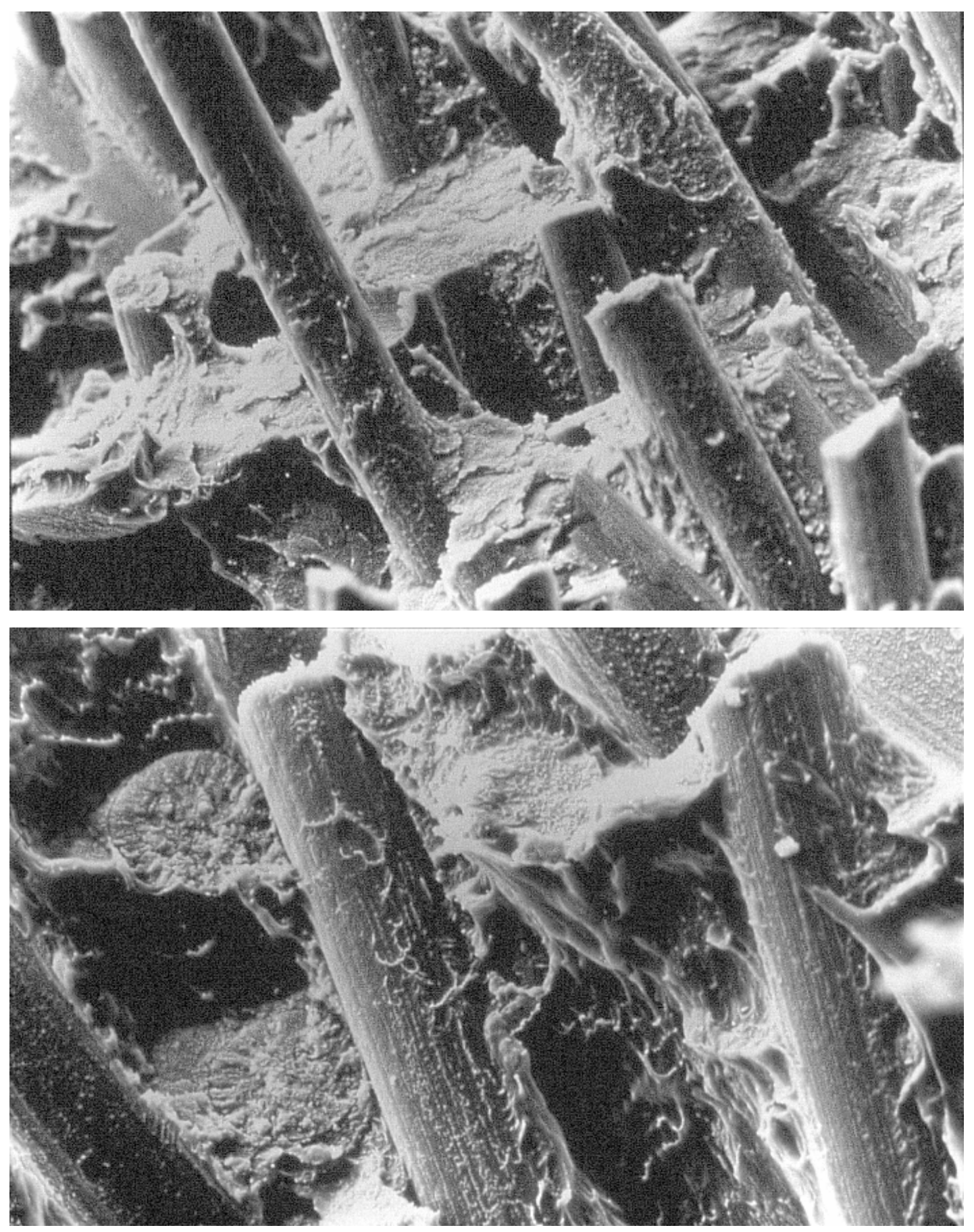

Figura 3. La matriz adhiere íntimamente a las fibras de carbono mojándolas. 
ciéndose un material que reúna los atributos positivos de ambas sin los defectos de ninguna. Las propiedades mecánicas de un material compuesto dependen en gran medida de la unión que se logre entre la matriz y las fibras (38).

Las posibilidades de estos materiales son ilimitadas y hoy día se está intentando aplicar este dominio al mundo de las prótesis articulares, entre otros. Lo más frecuente ha sido hasta el momento emplear las fibras de carbono $(32,39-41)$ o de poliaramida $(41,42)$, y como matriz resinas de polietileno (32), de poliacetal, de epoxi (43), o termoplásticos como la polisulfona $(40,41)$, la polieter-aril-cetona (PEAK), la poli-eter-cetona-cetona (PEKK) (44) o la poli-eter-eter-cetona (PEEK) $(41,45)$.

Ante los resultados cuestionables de otros materiales en la constitución de compuestos para superficies articulares protésicas $(32,33,43)$, se eligió la poli-eter-eter-cetona como matriz por su buen comportamiento químico $(46,47,48)$, su supuesta biocompatibilidad $(49,50)$, sus convenientes
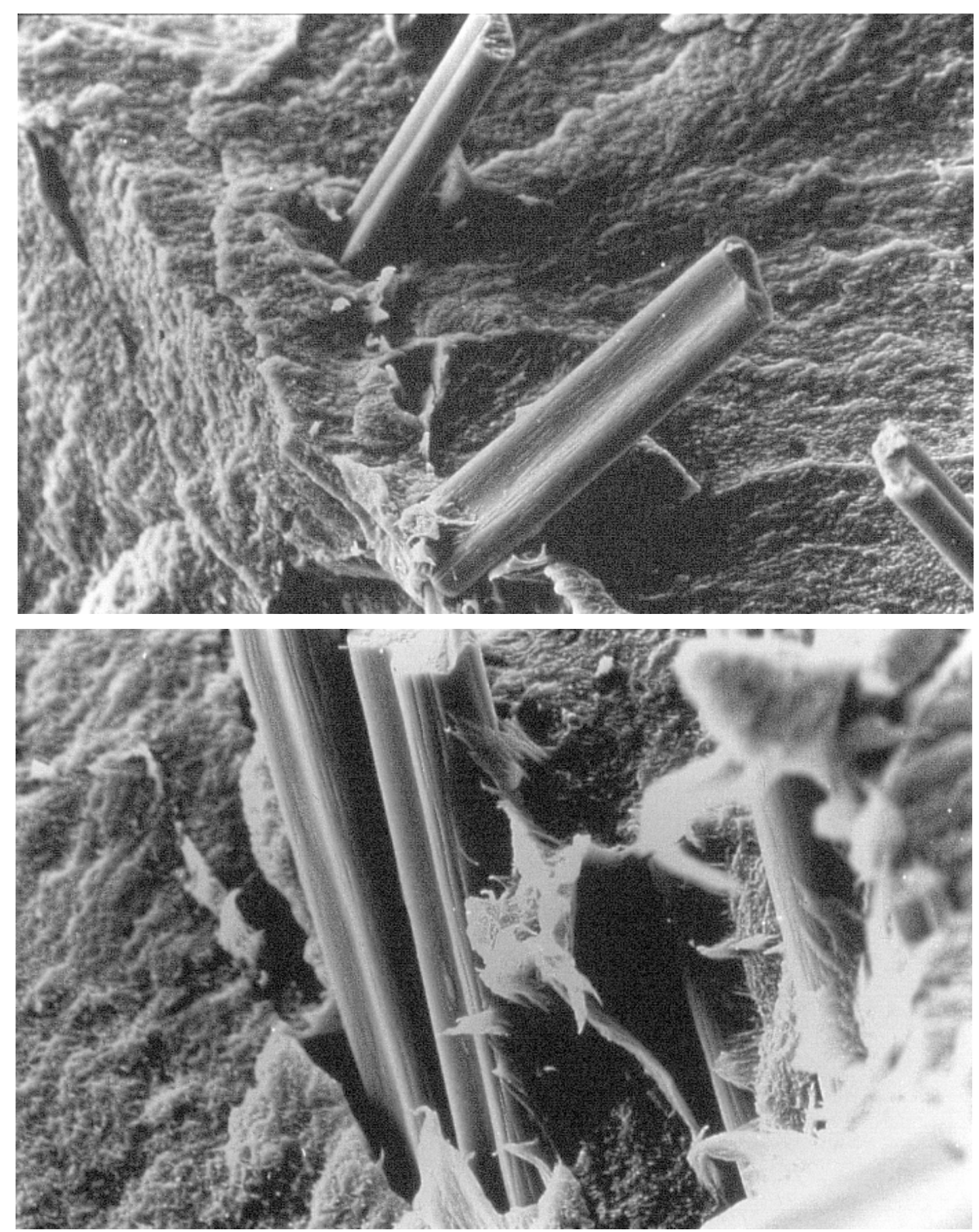

Figura 4. El UHMWPE no adhiere a las fibras no formando pues un auténtico material compuesto. 
propieda- des mecánicas (41), y la facilidad de su procesado. En efecto, el PEEK es un polímero semicristalino que funde a $343^{\circ} \mathrm{C}$ y que presenta una amplia gama de viscosidades. Se ha seleccionado la viscosidad más baja para trabajar el compuesto FC/PEEK mediante un proceso de extrusión, mezclandose íntimamente el PEEK en polvo y las fibras antes de la fusión (fig 3). Así se consigue una distribución regular de las fibras en la matriz, que las moja adhiriéndolas específicamente. Es un verdadero compuesto y no una mezcla que es lo que resulta ser en realidad el Poly II. El UHMWPE mantiene un comportamiento térmico viscoelástico, alcanzando un estado de goma blanda de viscosidad alta, siendo imposible mezclarla correctamente con las fibras. El mayor problema es pues la escasa resistencia de la unión entre fibras y matriz, no existiendo entre ellas adhesión específica (fig 4).

Esa magnífica compatibilidad mecánica entre las dos fases del material está probablemente en la base de su excelente comportamiento frente al desgaste, superior al del UHMWPE en los ensayos realizados.

Hay que resaltar que los valores de desgaste obtenidos con el método CMM son generalmente más altos que los conseguidos a través del registro de los pesos de los es-pecímenes a lo largo del experimento. Esto es debido a que este último sistema mide las pérdidas de peso mientras que el CMM lo hace respecto a cambios de volumen que incluyen el desgaste y la deformación por fluencia. Esta diferencia tiene lugar sobre todo en el primer millón de ciclos y no se incrementa con en aumento del número de ciclos, lo que ratifica que es debido a la fluencia más bien que a cualquier error significativo de esos métodos. Además, hay que notar que la medida del peso mide el desgaste de ambas superficies, la articular y la interfaz entre los dos componentes del cotilo, mientras que el CMM sólo registra las modificaciones de volumen a nivel de la superficie articular. En los acetábulos de material compuesto el desgaste ocurre también en los relieves en cremallera de su cara posterior, durante el proceso de asentamiento, y ésto se refleja en los valores de pérdida de peso comprobados durante el primer medio millón de ciclos.

Los resultados de los estudios realizados en cuanto a la biocompatibilidad del material utilizado coinciden con lo ya aportado por la literatura $(49,50)$. Evidentemente falta extrapolar todos ellos a la experiencia in vivo ya que son bien conocidas las limitaciones que tienen en este terreno los estudios en laboratorio, sobre modelos animales y sobre simuladores (3,37). Desde 2001 se llevan implantados 53 componentes acetabulares frente a cabezas de alúmina, en prótesis $\mathrm{ABG}$, por el grupo de estudio internacional, esperando al fin de este año 2003 para cerrar la fase de experimentación clínica y analizar los resultados.

\section{Conclusiones}

1. Los acetábulos de material compuesto FC/ PEEK demostraron una resistencia al desgaste superior a la de los d UHMWPE.

2. El desgaste de los elementos de compuesto después de 10 millones de ciclos es un $1 \%$ del de los de UHMWPE.

3. Los cotilos de UHMWPE muestran una tasa de penetración lineal de $66,41 \pm 12,64 \mu \mathrm{m}$ por millón de ciclos en tanto que la de los de FC/PEEK es de $1,72 \pm 1,36 \mu \mathrm{m}$ por millón de ciclos.

4. El elemento de compuesto se asienta en el componente metálico durante el primer $0,5 \mathrm{mi}-$ llón de ciclos.

5. El anillo de PEEK de bloqueo entre ambos componentes actuó satisfactoriamente durante los 10 millones de ciclos sin mostrar daño o aflojamiento del nucleo de compuesto insertado en el respaldo metálico.

6. Las cabezas de zirconio son compatibles con el cotilo de FC/PEEK, no observándose lesiones de las mismas durante los ensayos.

Nuestro agradecimiento a Howmedica por toda la ayuda prestada para realizar este trabajo.

\section{Bibliografía}

1. Mulroy WF, Estok DM, Harris WH. Total hip arthroplasty with use of so-called second-generation cementing techniques. J Bone Joint Surg 1995;77A:1845-1852.

2. Munuera L, García Cimbrelo E. La prótesis total de cadera cementada. Madrid: McGraw-HillInteramericana; 1989.

3. Wright TM, Goodman SB, eds. Implant wear: the future of total joint replacement. Rosemont, Illinois, American Academy of Orthopaedic Surgeons; 1996.

4. García Cimbrelo E, Munuera L. Early and late loosening of the acetabular cup after low-friction arthroplasty. J Bone Joint Surg 1992;74A:1119-1129.

5. Feller JA, Kay PR, Hodgkinson JP. Activity and socket wear in the Charnley low-friction arthroplasty. J Arthorplasty 1994;9:341-345. 
6. Schmalzried TP, Szuszczewicz ES, Northfield MR et al. Quantitative assessment of walking activity after total hip or knee replacement. J Bone Joint Surg 1998;80A:54-59.

7. Schmalzried TP, Callaghan JJ. Wear in total hip and knee replacements. J Bone Joint Surgery 1999;81A:115-136.

8. Howie DW. Tissue response in relation to type of wear particles around failed hip. J Arthroplasty 1990;5:337-348.

9. Amstutz, HC, Campbell P, Kossovsky N, Clarke IC. Mechanism and clinical significance of wear debris-induced osteolysis. Clin Orthop 1992;276:718.

10. Harris WH. The problem is osteolysis. Clin Orthop 1995;311:46-53.

11. McCoy TH, Salvati EA, Ranawat CS.A fifteen-year follow-up study of one hundred Charnley lowfriction arthroplasties. Orthop Clin North Am 1988; 19:467-476.

12. Shulte KR, Callaghan JJ, Kelley SS. The outcome of Charnley total hip arthroplasty with cement after a minimum twenty-year follow-up. J Bone Joint Surg 1993;75A:961-975.

13. Wroblewski BM. Fiteen to twenty-one year results of the Charnley low-friction arthroplasty. Clin Orthop 1986;211:30-35.

14. García Cimbrelo E. Prótesis de Charnley después de 10 años. En: Vidal J, Simon L, eds. Prótesis de cadera y rodilla. Barcelona: Masson SA; 1991, pp 41-47.

15. Judet $\mathbf{R}$, Judet J. Essais de réconstruction prothètique de la hanche après résection de la tête fémorale. J Chir 1949;65:17-24.

16. Waugh W. John Charnley, the man and the hip. New York: Springer Verlag; 1990.

17. Galante JO, Rostoker W. Wear in total hip prostheses. An experimental evaluation of candidate materials. Acta Orthop Scand 1973;145:5-46.

18. Sudmann E, Havelin LI, Lunda OD, Rait M. The Charnley vs the Christiansen total hip arthroplasty - a comparative clinical study. Acta Orthop Scand 1983;54:545-552.

19. Alho A, Soreide O, Bjersand A. Mechanical factors in loosening of Christiansen and Charnley arthroplasties. Acta Orthop Scand 1984;55:261-266.

20. Weber HG, Sthmer G. The trunnion bearing total hip prosthesis. En: Schaldach M, Hohmann D, eds. Advances in hip and knee technologies. New York: Springer Verlag; 1976, pp 203-210.

21. McKellop HA, Röstlund TV. The wear behavior of ion-implanted Ti-6Al-4V against UHMW polyethylene. J Biomed Mater Res 1990;24:14131425.

22. Sioshansi P. Ion implantation improves wear resistance of titanium. Orthopedics today 1990;10: 24-26.
23. Pappas MJ, Makris G, Buechel FF. Titanium nitride ceramic film against polyethylene: A 48 million cycle wear test. Clin Orthop 1995;317:64-70.

24. Weber B. Experience with the Metasul total hip bearing system. Clin Orthop 1996; 329S:S69-S77.

25. Schmalzried TP, Peters PC, Maurer BT, Bragdon CR, Harris WH. Long duration metal-on-metal total hip replacements with low wear of the articulating surfaces. J Arthroplasty 1996;11: 322331.

26. Boutin P, Christel P, Dorlot JM, et al. The use of dense alumina-alumina ceramic combination in total hip replacement. J Biomed Mater Res 1988;22:1203-1232.

27. Sedel L, Nizard RS, Kerboull L, et al. Aluminaalumina hip replacement in patients younger than 50 year old. Clin Orthop 1994;298:175-183.

28. Davidson JA, Schwartz G, Lynch G, Gir S. Wear, creep, and frictional heating of femoral implant articulating surfaces and the effect on long-term performance - Part II, friction, heating and torque. J Biomed Mater Res, Applied Biomaterials 1988;22A1:69-91.

29. Saikko V. Tribology of total replacement hip joints studied with new hip joints simulators and a materials screening apparatus. Acta Polytech Scand 1993; Mechanical Eng Series 110.

30. Rees DV, Basset DC. Crystallization of polyethylene at elevated pressures. J Polymer Science 1971;Part 2A,9:385-406.

31. Li S, Burstein AH. Ultra-high molecular weigth polyethylene: The material and its use in total joint implants. J Bone Joint Surg 1994;76A:1080-1090.

32. Wright TM, Fukubayshi T, Burstein AH. The effect of carbon reinforcement on contact area, contact pressure and time-dependent deformation in polyethylene tibial components. J Biomed Mater Res 1981; 15:719-730.

33. Wright TM, Rimmac CM, Faris PM, Bansal M. Analysis of surface damage ocurring in carbonfiberreinforced and plain polyethylene tibial components from posterior stabilized type total knee replacement. J Bone Joint Surg 1988;70A:1312-1327.

34. Lin R, Stark C. Summary report for composite insert/cup development. Report for Composite

Technology Transfer Program Package, Abril, 1997.

35. Mejia LC, Brierley TJ.A hip wear simulator for the evaluation of biomaterials in hip-arthroplasty components. Biomed Mat Eng 1994;4:259-271.

36. Paul J.Loading on normal hip and knee joints and on joint replacements. En: Schaldach M, Hohman $\mathrm{D}$, eds. Engineering in Medicine, Vol 2, Advances in Artificial Hip and Knne Joint

Technology. New York; Springer Verlag;1976, pp 5370.

37. McKellop HA, Clarke IC. Evolution and evaluation of materials-screening machines and jointsimulators 
in predicting in vivo wear phenomena. En: Ducheyne $P$, Hastings $G$, eds. Functional Behavior of Orthopaedic Biomaterials, Vol II, Applications. Boca Ratón; CRC Press;1984, pp 51-85.

38. Latour RA, Black J. Development of FRP composite structural biomaterials: Ultimate strength of the fiber/matrix interfacial bond in in vivo simulated environments. J Biomed Mat Res 1992;26: 593-606.

39. Pilliar RM, Blackwell R, Macnab I, Cameron HU. Carbon fiber-reinforced bone cement in orthopedic surgery. J Biomed Mat Res 1976;10:893-906.

40. Huettner W, Keuscher G, Nietert M. Carbon fibrereinforced polysulfone-thermoplastic composites. En: Ducheyne P, Van der Perre G, Aubert AE, eds. Biomaterials and Biomechanics 1983. Amsterdam; Elsevier; 1984, pp 167-172.

41. Skinner HB. Composite technology for total hip arthroplasty. Clin Orthop 1988;235:224-236.

42. Henderson JD, Mullarky RH, Ryan DE. Tissue biocompatibility of kevlar aramid fibers and polymethylmethacrylate composites in rabbits. J Biomed Mat Res 1987;21:59-64.

43. Boenisch B. Five year functionality study of carbon fibre reinforced acetabular implants in dogs. En: Heimke G, Soltése V, Lee AJC, eds. Advances in Biomaterials, Vol 9. Amsterdam; Elsevier; 1990, pp 391-396.
44. Moore $R$, Beredjiklian $P$, Rhoad $R$ et al. A comparison of the inflammatory potential of particulates derived from two composite materials. J Biomed Mat Res 1997; 34:137-147.

45. Akay M, Aslan N. Numerical and experimental stress analysis of a polymeric composite hip joint prosthesis. J Biomed Mat Res 1996; 31:167-182.

46. Cogswell FN, Hopprich M. Environmental resistance of carbon fiber-reinforced poly ether ketone. Composites 1983; 14:251-253.

47. Stober EJ, Seferis JC, Keenan JD. Characterization and exposure of poly ether ether ketone (PEEK) to fluid environments. Polymer 1984; 25:1845-1852.

48. Ma C, Yur S. Environmental effects on the water absorption and mechanical properties of carbon fiber reinforced PPS and PEEK composites. Polym Eng Sci 1991; 31:34-39.

49. Williams DF, McNamara A, Turner RM. Potential of poly ether ether ketone (PEEK) carbon-fiberreinforced PEEK in medical applications. J Mater Sci Let 1987; 6:188-190.

50. Wenz LM, Merritt K, Moet A, Steffe AD. In vitro biocompatibility of poly ether ether ketone and polysulfone composites. J Biomed Mater Res 1990; 24:207-215. 